\title{
Regulation of Carbohydrate Utilization in Clostridium pasteurianum
}

\author{
By WILFRID J. MITCHELL, ${ }^{1 *}$ M. SADEGH ROOHI, ${ }^{1}$ \\ MARTIN J. MOSELY ${ }^{2}$ AND IAN R. BOOTH ${ }^{2}$ \\ ${ }^{1}$ Department of Brewing \& Biological Sciences, Heriot-Watt University, Chambers Street, \\ Edinburgh EHI $1 \mathrm{HX}, \mathrm{UK}$ \\ ${ }^{2}$ Department of Microbiology, University of Aberdeen, Marischal College, \\ Aberdeen $A B 91 A S, U K$
}

(Received 16 June 1986; revised 5 August 1986)

\begin{abstract}
Clostridium pasteurianum is capable of fermentative growth on a number of carbohydrate compounds. Several, including glucose, fructose and sorbitol, are accumulated via a phosphoenolpyruvate-dependent phosphotransferase system (PTS), while the uptake of galactose and gluconate is protonmotive-force-dependent. We have examined the utilization of these substrates by cultures of $C$. pasteurianum growing on carbohydrate mixtures to determine whether the organism displays preferences for one carbon source over the others; such a preference may indicate the operation of specific mechanisms for regulation of carbohydrate metabolism. In most cases the carbohydrates were co-metabolized. Glucose was utilized together with fructose, gluconate and galactose, although galactose appeared to be favoured over glucose. This preference was not due to repression of synthesis of the glucose PTS. On the other hand, glucose prevented induction of the sorbitol PTS, and led to strong inhibition of sorbitol utilization in uninduced cells. Pre-adaptation of cells to growth on sorbitol counteracted the inhibition by glucose resulting in utilization of glucose and sorbitol at approximately equal rates.
\end{abstract}

\section{INTRODUCTION}

Bacteria display a remarkable capacity to regulate synthesis of enzyme systems with varying growth conditions. In addition to their capacity to repress enzyme systems which are not essential to growth, they often display preferences for one carbon source over another. In Escherichia coli, glucose is the preferred carbon source and when present it both prevents the induction of operons concerned with the utilization of other compounds and inhibits the activity of pre-induced metabolic systems for other carbon sources. The phenomenon by which glucose inhibits metabolism of other substrates has been referred to variously as the glucose effect or catabolite repression (Magasanik, 1970). Inhibition of sugar metabolism by glucose has been shown to be mediated principally by the phosphoenolypyruvate (PEP)-dependent phosphotransferase system (PTS) which transports glucose into the cell (Postma \& Lengeler, 1985). The control mechanism has at least two components; inhibition of transport of the sugar (inducer exclusion) and regulation of adenylate cyclase activity. Thus in the presence of glucose, the intracellular concentration of both cAMP and inducers of enzyme systems is decreased. Since both are required for expression of many inducible regulons, enzyme synthesis is extremely sensitive to glucose.

Although Gram-positive bacteria are also known to exhibit catabolite repression (Priest, 1984), the mechanisms involved in regulation of gene expression in these organisms have only

\footnotetext{
Abbreviations: PEP, phosphoenolpyruvate; PTS, phosphotransferase system; RCM, Reinforced Clostridial Medium.
} 
recently begun to be elucidated. Studies in this area concerning clostridia have concentrated on the regulation both of the synthesis of components of the cellulase complex and of the utilization of the products of cellulose breakdown (reviewed in Booth \& Mitchell, 1987). The emerging consensus is that, whilst in some species there is evidence for catabolite repression by a range of sugars, in many instances carbohydrate co-metabolism occurs. As part of our investigation of the role of the PEP-dependent phosphotransferase in the metabolism of carbohydrates (Mitchell \& Booth, 1984), we have examined carbohydrate co-metabolism in Clostridium pasteurianum. The aim of this work was to identify interactions which would form the basis of later studies of metabolic and genetic control of carbohydrate utilization in this species.

\section{METHODS}

Organism and growth conditions. Clostridium pasteurianum NCIB 9486 was maintained as a spore suspension at $4{ }^{\circ} \mathrm{C}$. A small sample $(0.5-1.0 \mathrm{ml})$ was treated at $80^{\circ} \mathrm{C}$ for $10 \mathrm{~min}$, inoculated into $20 \mathrm{ml}$ Reinforced Clostridial Medium (RCM) (Oxoid), and incubated for $24 \mathrm{~h}$ at $37^{\circ} \mathrm{C}$. A sample of the culture was then inoculated into $100 \mathrm{ml}$ of synthetic RCM containing the required carbon source at $0.5 \%(\mathrm{w} / \mathrm{v})$. Anaerobic conditions were maintained by evacuation of the culture headspace and replacement with nitrogen. After incubation overnight at $37^{\circ} \mathrm{C}$, the cells were harvested by centrifugation at $4^{\circ} \mathrm{C}$ and washed with growth medium lacking carbon source but containing $0 \cdot 1 \%(\mathrm{w} / \mathrm{v}) \mathrm{L}$-cysteine hydrochloride and $10 \mathrm{~mm}$-sodium pyruvate. A sample of the final suspension, calculated to give a suitable initial cell density, was then inoculated into the experimental culture. The competing substrate was either present initially or was added after growth had commenced. Growth was monitored by measuring optical density at $650 \mathrm{~nm}$. The above protocol was followed in all experiments except those involving sorbitol, in which a defined mineral salts medium (Mitchell \& Booth, 1984) was used in place of synthetic RCM.

Throughout the experimental growth phase, $1 \mathrm{ml}$ samples were taken from the culture, cells removed by centrifugation in an Eppendorf centrifuge, and the carbohydrate concentration in the supernatants assayed. In experiments involving both glucose and fructose, the sugars were assayed using a glucose/fructose assay kit (Boehringer-Mannheim); otherwise glucose was assayed using Sigma assay kit no. 510. Galactose, gluconate and sorbitol concentrations were assayed using kits obtained from Boehringer-Mannheim. All experiments illustrated are representative of two or three similar experiments.

Assay of sugar uptake. This was done as described by Booth \& Morris (1975, 1982).

Assay of sorbitol PTS activity. Cells growing exponentially in defined mineral salts medium containing excess $(2 \%, \mathrm{w} / \mathrm{v})$ carbon source were harvested and washed with $50 \mathrm{~mm}$-potassium phosphate buffer, $\mathrm{pH} 7 \cdot 0$, containing $0.1 \%(\mathrm{w} / \mathrm{v})$ L-cysteine hydrochloride. Activity of the sorbitol PTS was measured by following the rate of phosphorylation of $\left[{ }^{14} \mathrm{C}\right]$ sorbitol in cells permeabilized by treatment with toluene (Mitchell \& Booth, 1984; Mitchell et al., 1985). Cell suspensions were supplemented with $5 \mathrm{mM}-\mathrm{MgCl}_{2}$ and $0.5 \mathrm{mM}-\mathrm{PEP}$, and after a $5 \mathrm{~min}$ equilibration period at $37^{\circ} \mathrm{C},\left[{ }^{14} \mathrm{C}\right]$ sorbitol $\left[1 \mathrm{mCi} \mathrm{mmol}^{-1}\left(37 \mathrm{MBq} \mathrm{mmol}{ }^{-1}\right)\right]$ was added to a concentration of $0.1 \mathrm{mM}$. Labelled hexitol phosphate was isolated by precipitation in ethanolic $\mathrm{BaBr}_{2}$ as described by $\mathbf{M i t c h e l l} \&$ Booth (1984).

Materials. $\mathrm{D}(+)$ Glucose, $\mathrm{D}(-)$ fructose, $\mathrm{D}(+)$ sorbitol, $\mathrm{D}(+)$ galactose and potassium gluconate were obtained from Sigma. Other chemicals were of the highest purity available commercially. D- $\left[\mathrm{U}-{ }^{14} \mathrm{C}\right]$ Sorbitol, methyl $\alpha-\mathrm{D}-$ $\left[\mathrm{U}-{ }^{14} \mathrm{C}\right] \mathrm{glucopyranoside}$ and $\mathrm{D}-\left[1{ }^{-14} \mathrm{C}\right] \mathrm{galact}$ ose were purchased from Amersham.

\section{RESULTS AND DISCUSSION}

\section{Utilization of glucose and other PTS sugars}

When cells of $C$. pasteurianum pre-grown on glucose were used to inoculate medium containing both glucose and sorbitol, the hexitol was not appreciably removed from the medium until the glucose had been extensively depleted (Fig. 1a); however no diauxie was observed in the growth curve. Glucose utilization was not affected by the presence of sorbitol (data not shown). If, on the other hand, cells were pre-adapted to sorbitol, the two substrates were cometabolized and glucose merely reduced the rate of hexitol consumption to a level comparable to that of glucose itself (Fig. $1 b$ ). Nevertheless, despite the fact that the rate of utilization of glucose and sorbitol was similar, experiments following the incorporation of ${ }^{14} \mathrm{C}$-labelled carbohydrates have indicated a strong preference for glucose (Mitchell et al., 1985), demonstrating that carbon derived from glucose is preferentially incorporated into cellular material.

The sorbitol PTS has been reported to be constitutive in $C$. pasteurianum, but activity in glucose-grown cells was nonetheless extremely low (Booth \& Morris, 1982). We therefore reexamined the activity of the sorbitol PTS in cultures growing exponentially on glucose, 


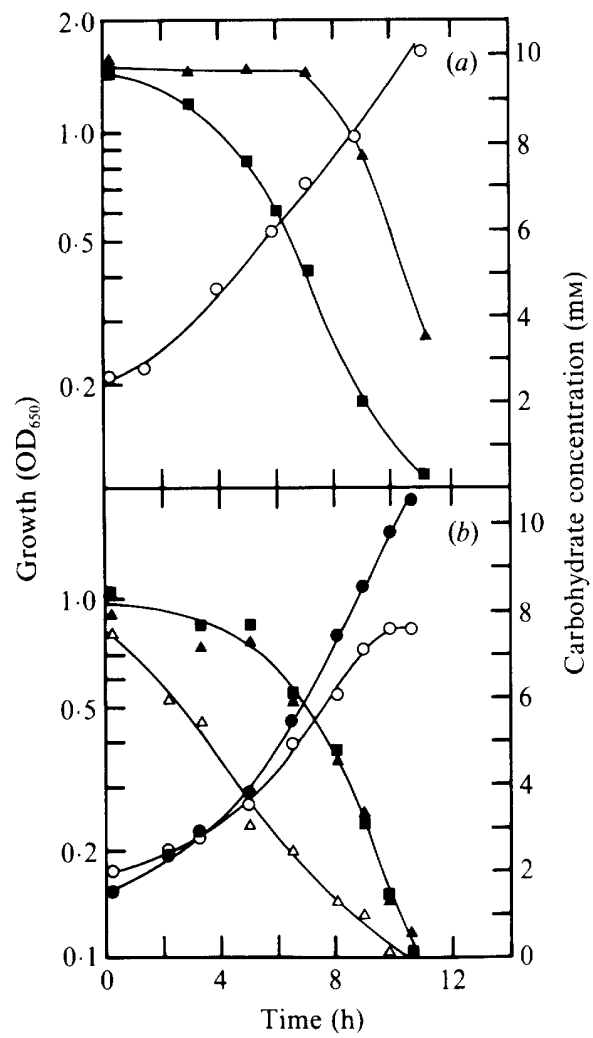

Fig. 1

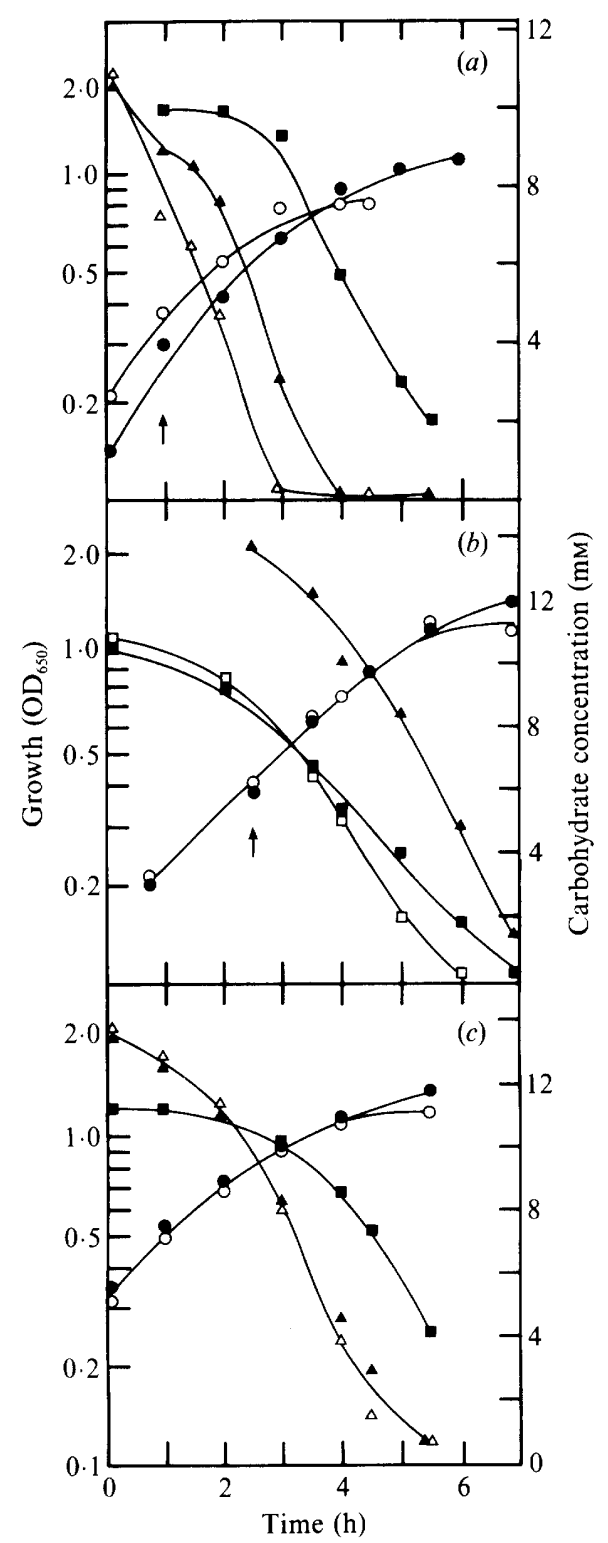

Fig. 2

Fig. 1. Effect of glucose on sorbitol utilization by $C$. pasteurianum. (a) Cells pregrown on glucose and inoculated into medium containing glucose plus sorbitol. $\mathrm{O}, \mathrm{OD}_{650} ; \boldsymbol{\square}$, glucose concentration in medium; $\boldsymbol{\Lambda}$, sorbitol concentration in medium. $(b)$ Cells pregrown on sorbitol and inoculated into medium containing sorbitol only $(O, \triangle)$ or sorbitol plus glucose $(\mathbf{O}, \boldsymbol{\Delta}, \mathbf{0}), 0, \mathbf{O}, \mathrm{OD}_{650}, \triangle, \boldsymbol{\Delta}$, sorbitol concentration in medium; $\boldsymbol{\square}$, glucose concentration in medium.

Fig. 2. Utilization of glucose and non-PTS sugars by $C$. pasteurianum. (a) Effect of glucose on gluconate utilization. $\bigcirc, \triangle$, Control culture with gluconate as sole carbon source: $O, \mathrm{OD}_{650} ; \triangle$, gluconate concentration in medium. $, \boldsymbol{\Delta}, \boldsymbol{\square}$, Culture with glucose added at the time indicated by the arrow: $\boldsymbol{O}$, $\mathrm{OD}_{650} ; \mathbf{\Lambda}$, gluconate concentration in medium; $\boldsymbol{\square}$, glucose concentration in medium. $(b)$ Effect of galactose on glucose utilization. $\bigcirc, \square$, Control culture with glucose as sole carbon source: $O, \mathrm{OD}_{650}$; $\square$, glucose concentration in medium. $, \mathbf{A}, \boldsymbol{\square}$, Culture with galactose added at the time indicated by the arrow:, $\mathrm{OD}_{650} ; \boldsymbol{\square}$, glucose concentration in medium; $\boldsymbol{A}$, galactose concentration in medium. $(c)$ Effect of glucose on galactose utilization. $O, \triangle$, Control culture with galactose as sole carbon source: $\mathrm{O}, \mathrm{OD}_{650} ; \triangle$, galactose concentration in medium. $, \boldsymbol{\Delta}, \mathbf{Q}$, Culture containing both glucose and galactose:, $\mathrm{OD}_{650} ; \boldsymbol{\Delta}$, galactose concentration in medium; $\boldsymbol{\square}$, glucose concentration in medium. 
sorbitol and a mixture of the two carbon sources, by following phosphorylation of $\left[{ }^{14} \mathrm{C}\right]$ sorbitol by cells permeabilized with toluene (Mitchell \& Booth, 1984). The rate of sorbitol phosphorylation by cells grown on sorbitol was in the range $1.9-2.2 \mathrm{nmol} \mathrm{min}{ }^{-1}$ (mg dry wt cells $)^{-1}$, some 20 -fold higher than cells grown on glucose. Cells grown on a mixture of glucose and sorbitol exhibited the uninduced level of sorbitol PTS activity (data not shown). The implication of these results is that, in uninduced cells, sorbitol is effectively excluded in the presence of glucose. Partial inhibition of sorbitol uptake by glucose and the analogue methyl $\alpha-$ glucoside has in fact been demonstrated in fully induced cells (Booth \& Morris, 1982; Mitchell et al., 1985). The extent of the inhibition is now known to be dependent on the ability of the sorbitol PTS to compete with the glucose PTS for a common energy pool (M. S. Roohi \& W. J. Mitchell, unpublished), and is thus related to the level of sorbitol transport activity in the cell.

C. pasteurianum did not discriminate between glucose and fructose as growth substrates. Thus, on addition of one of these sugars to cells growing on the other, both were metabolized simultaneously. No change in the growth rate was observed when the second sugar was added, but rather there was an adjustment of the rate of utilization of the sugar initially present (data not shown). These results were consistent with constitutive phosphotransferase systems for glucose and fructose in C. pasteurianum (Booth \& Morris, 1982), and indicated that, unlike other organisms (Kornberg, 1973; Amaral \& Kornberg, 1975; Dills et al., 1980), C. pasteurianum has no sophisticated regulatory mechanism allowing differentiation between these two carbon sources.

\section{Utilization of glucose and non-PTS sugars}

Addition of glucose to a culture of $C$. pasteurianum pre-induced for utilization of gluconate resulted in a marked decrease in the rate of gluconate consumption, without affecting the growth rate. In the first $2 \mathrm{~h}$ after its addition, the utilization of glucose was very slow and was only evident through its sparing effect on gluconate uptake, while subsequently the rate of metabolism of both substrates increased (Fig. $2 a$ ). On the other hand, gluconate had no discernible effect on the rate of glucose utilization, although once again co-metabolism of the substrates was observed (data not shown). These results were consistent with the earlier observation that induction of the gluconate transport system of $C$. pasteurianum was not prevented by the presence of glucose (Booth \& Morris, 1975). It has, however, been found that glucose strongly inhibits incorporation of $\left[{ }^{14} \mathrm{C}\right]$ gluconate into cellular material in growing cells (Booth, 1976). It must be concluded, therefore, that glucose exerts some metabolic control over gluconate incorporation at some point subsequent to the transport step, possibly at the level of gluconeogenesis. The effects of fructose on gluconate utilization were similar to those illustrated for glucose (data not shown).

The consumption of glucose and galactose, either singly or in the presence of each other, is shown in Fig. 2(b,c). The two carbon sources were utilized simultaneously, although there was a distinct preference for galactose over glucose. Thus addition of galactose to a culture growing on glucose resulted in a small, but significant sparing of glucose, and galactose was utilized immediately. On the other hand, glucose was used only slowly when added to cells adapted to galactose. This preference for galactose was reflected in the rate of incorporation of $\left[{ }^{14} \mathrm{C}\right]$ glucose, which was reduced by $80 \%$ in the presence of galactose (Booth, 1976). The effects of galactose could not be attributed to lack of induction of the glucose transport system. Addition of glucose to cells growing on galactose resulted in an increase in the glucose PTS to a level similar to that found in glucose-grown cells, as indicated by the rate of uptake of methyl $\alpha$ glucoside (Fig. 3). Glucose had no significant effect on galactose transport activity, which was rapidly induced on addition of galactose to glucose-grown cells and was little affected by addition of glucose to galactose-grown cells.

Preference of $C$. pasteurianum for galactose over glucose was an unexpected finding, since in most bacteria PTS sugars appear to be preferred to non-PTS substrates (Saier, 1977). The basis of galactose preference is not understood, but it is likely that regulation of carbon flux arises from one of three effects: first, inherent differences in the kinetics of galactose and glucose transport; second, regulation by galactose of glucose influx; and third, preferential incorporation of carbon derived from galactose into cellular material. 


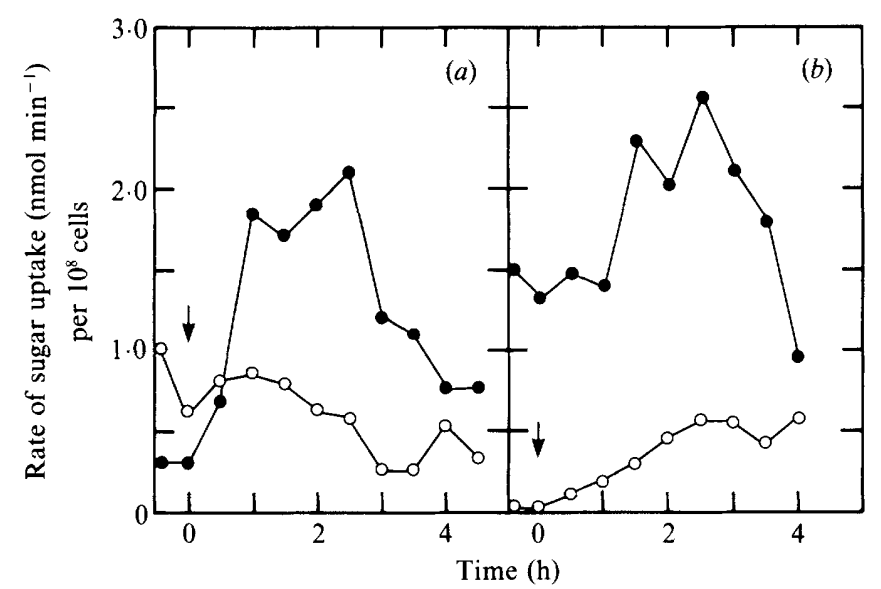

Fig. 3. Uptake of galactose and methyl $\alpha$-glucoside by $C$. pasteurianum. Cells were prepared by growing on either $(a)$ galactose or $(b)$ glucose, and inoculated into medium containing the same carbohydrate. At $t=0$, the second carbohydrate was added. At the times indicated, samples were taken from the growing culture and carbohydrate uptake rates measured as described in Methods. $O$, Rate of galactose uptake;, rate of methyl $\alpha$-glucoside uptake.

\section{Conclusion}

We have examined utilization of glucose, sorbitol, fructose, gluconate and galactose by growing cells of $C$. pasteurianum. The carbohydrates examined reflect different routes of transport and subsequent metabolism, which have different energy yields and demands, and accordingly significant preference for the most 'energy-efficient' carbon source was expected. The PEP-dependent phosphotransferase system did not play a major role in regulating the pattern of carbohydrate utilization as it does in enteric bacteria. C. pasteurianum co-metabolized glucose and the non-PTS substrates galactose and gluconate, and while regulation was observed in carbohydrate mixtures it appeared to occur at the level of carbon flux. Induction of metabolism of the PTS substrate sorbitol was, however, prevented in the presence of glucose. The mechanism of regulation of sorbitol metabolism by glucose has been shown to involve inhibition of sorbitol accumulation by the cell (Booth \& Morris, 1982; Mitchell et al., 1985), and a more extensive description of the mechanism of inhibition of sorbitol uptake by glucose will be presented elsewhere (M. S. Roohi \& W. J. Mitchell, unpublished work).

It may also be significant that there was little relationship between the rates of carbon consumption and growth, and this suggests that the growth rate of $C$. pasteurianum may not be limited by the rate of energy production.

We are grateful to Professor J. G. Morris for helpful discussions during the initial phase of this work. The work was supported in part by a research grant from the SERC (to I.R. B.).

\section{REFERENCES}

AMARAL, D. \& KornberG, H. L. (1975). Regulation of fructose uptake by glucose in Escherichia coli. Journal of General Microbiology 90, 157-168.

BоOTH, I. R. (1976). Studies on the cell membrane of Clostridium pasteurianum. PhD thesis, University of Wales, UK.

BoоTH, I. R. \& Mitchell, W. J. (1987). Sugar transport and metabolism in the clostridia. In Sugar Transport and Metabolism in Gram-positive Bacteria. Edited by J. Reizer \& A. Peterkofsky. Chichester: Ellis Horwood (in the Press).

Booth, I. R. \& Morris, J. G. (1975). Proton-motive force in the obligately anaerobic bacterium Clostridium pasteurianum: a role in galactose and gluconate uptake. FEBS Letters 59, 153-157.

BooTh, I. R. \& Morris, J. G. (1982). Carbohydrate transport in Clostridium pasteurianum. Bioscience Reports 2, 47-53.

Dills, S. S., Apperson, A., Schmidt, M. R. \& Saier, M. H., JR (1980). Carbohydrate transport in bacteria. Microbiological Reviews 44, 385-418.

KorNBERG, H. L. (1973). Fine control of sugar uptake by Escherichia coli. Symposium of the Society for Experimental Biology 27, 217-240. 
MAGASANIK, B. (1970). Glucose effects: inducer exclusion and repression. In The Lactose Operon, pp. 189219. Edited by J. R. Beckwith \& D. Zipser. Cold Spring Harbor, New York: Cold Spring Harbor Laboratory.

MitChell, W. J. \& Booth, I. R. (1984). Characterization of the Clostridium pasteurianum phosphotransferase system. Journal of General Microbiology 130, 2193-2200.

Mitchell, W. J., ROOHI, M. S. \& MORRISON, D. (1985). Regulation of sorbitol metabolism in Clostri- dium pasteurianum. Biochemical Society Transactions 13, 936-937.

Postma, P. W. \& Lengeler, J. W. (1985). Phosphoenolpyruvate : carbohydrate phosphotransferase system of bacteria. Microbiological Reviews 49, 232-269.

PRIEST, F. G. (1984). Extracellular Enzymes. Wokingham: Van Nostrand Reinhold (UK).

SAIER, M. H., JR (1977). Bacterial phosphoenolpyruvate : sugar phosphotransferase systems: structural, functional and evolutionary interrelationships. $\mathrm{Bac}$ teriological Reviews 41, 856-871. 